\title{
Prediction of RF-EMF exposure levels in large outdoor areas through car- mounted measurements on the enveloping roads
}

Authors: Sam Aerts ${ }^{\mathrm{a}, *}$, Wout Joseph ${ }^{\mathrm{a}}$, Myron Maslanyj ${ }^{\mathrm{b}}$, Darren Addison ${ }^{\mathrm{b}}$, Terry Mee ${ }^{\mathrm{b}}$, Loek Colussi ${ }^{\mathrm{c}}, \mathrm{Jos}_{\mathrm{Kamer}}^{\mathrm{c}}$, and John Bolte ${ }^{\mathrm{d}}$

\section{Affiliations:}

${ }^{a}$ Department of Information Technology, Ghent University/iMinds, G. Crommenlaan 8, B-9050 Ghent, Belgium

${ }^{b}$ Centre for Radiation, Chemical and Environmental Hazards, Public Health England, Chilton, Didcot, Oxon OX11 ORQ, United Kingdom

${ }^{c}$ Radiocommunications Agency Netherlands, Piet Mondriaanlaan 54, 3812GV Amersfoort, The Netherlands

${ }^{d}$ Centre for Sustainability, Environment and Health, National Institute for Public Health and Environment (RIVM), PO Box 1, 3720 BA Bilthoven, The Netherlands

*Corresponding author: sam.aerts@intec.ugent.be

Keywords: Radiofrequency electromagnetic fields; RF-EMF; mobile measurements; kriging; environmental exposure; epidemiology 


\section{Introduction}

Although ambient levels of radiofrequency (RF) electromagnetic fields (EMF) generally encountered in everyday life are well below established limits (ICNIRP, 1998) and thus not conclusively linked to adverse health effects (due to heating), there is a concern that long-term exposure at low levels may be associated with various non-specific physical symptoms (Baan et al., 2011; Baliatsas et al., 2015) and ecological effects on fauna and flora (Cucurachi et al., 2013).

A large-scale assessment of the effects of RF-EMF on health or on ecology requires the quantification of the ambient RF-EMF levels over areas too vast to cover with conventional measurement methods, i.e., personal exposimeter measurement surveys (Bolte and Eikelboom, 2012; Frei et al., 2009, 2010; Joseph et al., 2010a; Viel et al., 2009) or spot measurements (Joseph et al; 2010b) potentially extended with an interpolation scheme (Aerts et al., 2013a,b). Furthermore, RF exposure modelling, using software packets such as NISmap (Bürgi et al., 2010 Beekhuizen et al., 2013), requires accurate and upto-date base station data and a detailed map of the locations and dimensions of the buildings in the assessed area, which is not always available.

However, recently, various mobile measurement systems have been tested (Bolte et al., 2016; Estenberg and Augustsson, 2014; Tell and Kavet, 2014). For example, Bolte et al. (2016) have shown that, although initially built with the purpose of spectrum regulation (CFRS Ltd., 2009; Schiphorst and Slump, 2010), the mobile measurement systems used by the Radiocommunications Agency in the Netherlands, which consists of spectrum analysers with external antennas mounted on cars, are capable of accurately measuring RF exposure over large geographic areas in a relatively short time, potentially making them a suitable tool for large-scale RF-EMF exposure assessment. They found that exposure assessments through car-mounted measurements are at least similar in quality to assessments involving exposure modelling and better than those involving body worn exposimeter data due to the absence of the body shielding effect.

This study continues the effort of Bolte et al. (2016) and investigates, for the first time, the RF-EMF exposure related information that can be deduced for an arbitrary geographical area based on measurements on the roads encircling the area. To achieve this, we interpolated the RF exposure in five distinct (sub)urban areas using car measurement data collected along the (semi-)closed loops encircling the areas and validated the results with analogous measurements on a street grid within the area. The various outcomes helped to define the characteristic traits of an area (size and shape, and building characteristics) for this type of modelling methodology to offer valid results. Then we determined to what extent the interpolation can be improved by adding inner-area measurement samples, thus extending the methodology for larger and less ideal areas. 


\section{Materials and methods}

\subsection{Measurements}

For this study, we used measurements reported in a previous study (Bolte et al., 2016) (in particular, the "RFeye left" measurements).

Our data are derived from measurements at two frequency bands for mobile-phone downlink communication (i.e., signals from base stations to mobile phones) using the Global System for Mobile Communications (GSM) standard: GSM900 (925-960 MHz) and GSM1800 (1805-1880 MHz). The measurements were performed in five geographical areas in two cities (Cambridge, the United Kingdom, during the period 14th-16th May 2013, and Amersfoort, the Netherlands, on 22nd October 2013), the areas ranging in size between 0.5 and $2.5 \mathrm{~km}^{2}$ (Table 1). The measurement system consisted of an antenna mounted on a car rooftop connected to an RFeye measurement unit, comprising an integrated spectrum analyser with built-in GPS tracker of type Adapt AD-850 (Adapt Mobile Ltd., London, UK) and a data storage facility (CFRS Ltd., Cambridge, UK). The measurement uncertainty of the RFeye unit is $2.5 \mathrm{~dB}$.

\begin{tabular}{lccccccc}
\hline Area & $\boldsymbol{A}$ & $\boldsymbol{P}$ & $\boldsymbol{R C}$ & $\boldsymbol{n}_{\text {inner }}$ & $\boldsymbol{n}_{\text {ring }}$ & $\boldsymbol{d}_{\text {area }}$ & $\boldsymbol{d}_{\text {inner }}$ \\
Amersfoort-City & $0.63 \mathrm{~km}^{2}$ & $3.2 \mathrm{~km}$ & $79 \%$ & 90 & 103 & $139 \mathrm{~m}$ & $155 \mathrm{~m}$ \\
Amersfoort-Industrial & $1.08 \mathrm{~km}^{2}$ & $4.7 \mathrm{~km}$ & $63 \%$ & 116 & 141 & $155 \mathrm{~m}$ & $157 \mathrm{~m}$ \\
Amersfoort-Residential & $0.54 \mathrm{~km}^{2}$ & $4.5 \mathrm{~km}$ & $100 \%$ & 187 & 168 & $88 \mathrm{~m}$ & $95 \mathrm{~m}$ \\
Cambridge-City & $2.44 \mathrm{~km}^{2}$ & $6.5 \mathrm{~km}$ & $100 \%$ & 145 & 278 & $260 \mathrm{~m}$ & $307 \mathrm{~m}$ \\
Cambridge-Residential & $0.91 \mathrm{~km}^{2}$ & $5.0 \mathrm{~km}$ & $100 \%$ & 177 & 184 & $132 \mathrm{~m}$ & $142 \mathrm{~m}$ \\
\hline Table I: Area statistics: A = area bounded by the ring route, $P=$ perimeter of the ring route (if not closed, the ring route was \\
artificially closed to calculate A and P), RC = ringclosedness, nimner = number of inner data points, nring = number of ring data \\
points, darea = average minimum distance between inner area and ring, and dinner = average minimum distance between inner \\
data points and ring (distances are calculated between grid tile centres).
\end{tabular}

In both cities, a number of circular loops were defined; three in Amersfoort (one closed ring) (Figure 1 and Table 1), and two in Cambridge (both closed rings) (Figure 2 and Table 1). The car-mounted measurement system was set up to sample the power density in the two GSM frequency bands along these (ring) routes, as well as along an additional (inner) route selected within each enveloped area (Figures 1-2), while maintaining a maximum speed of $30 \mathrm{~km} / \mathrm{h}$. The RFeye performed 8 full sweeps every $2 \mathrm{~s}$, and was set to record in standard instantaneous peak mode as used by the Radiocommunications Agency (RCA) during spectrum regulation measurements, which means that it retains the maximum value out of the 8 sweeps (Bolte et al., 2016). The circular routes were driven at least twice (in both directions), while the inner route was covered only once. 


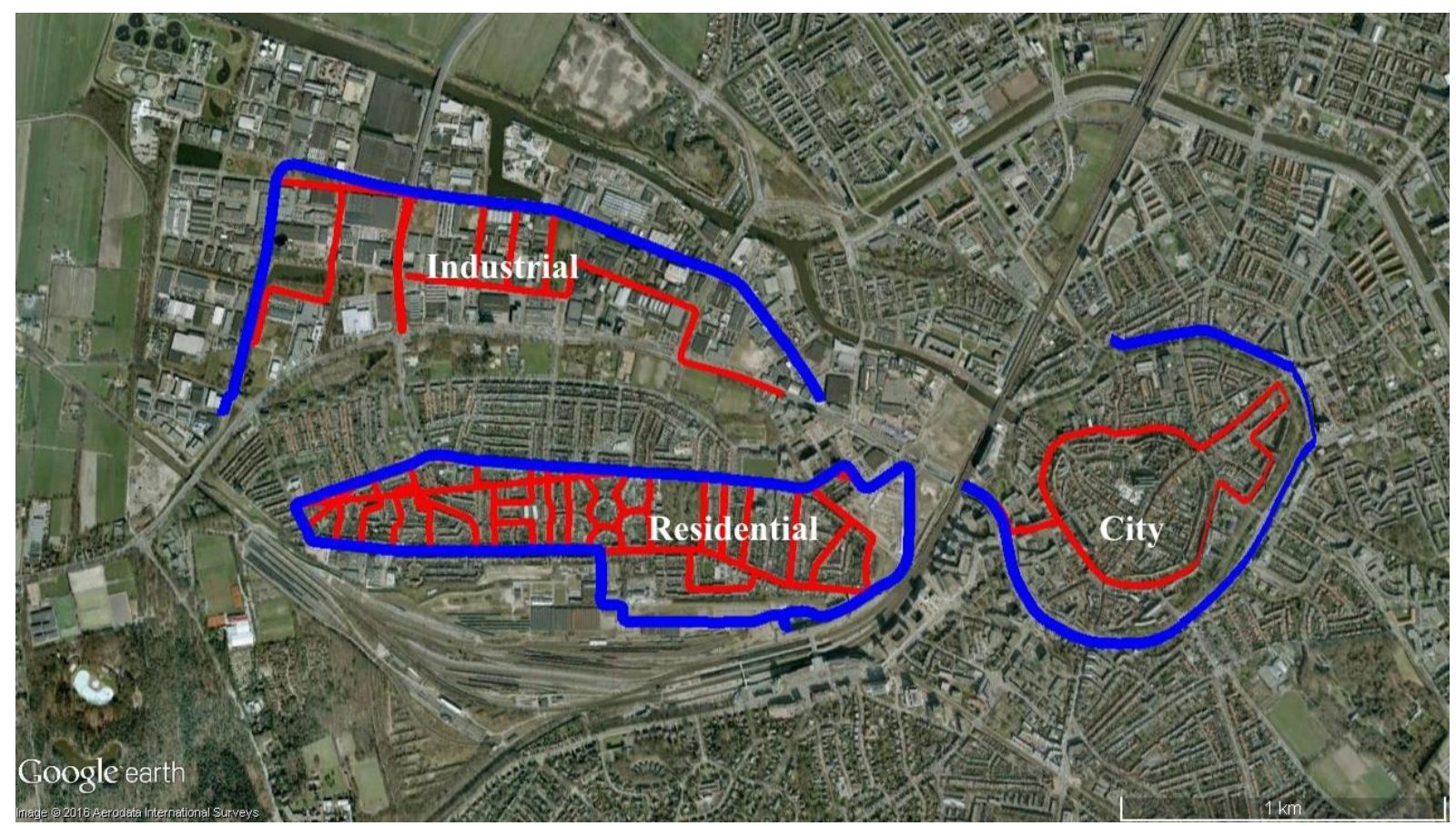

Figure 1. Amersfoort area. Three regions in Amersfoort, The Netherlands, with car measurements along ring (blue) and inner (red) roads. "Inner" measurements outside the area demarcated by the ring road were removed from the inner data set (I).

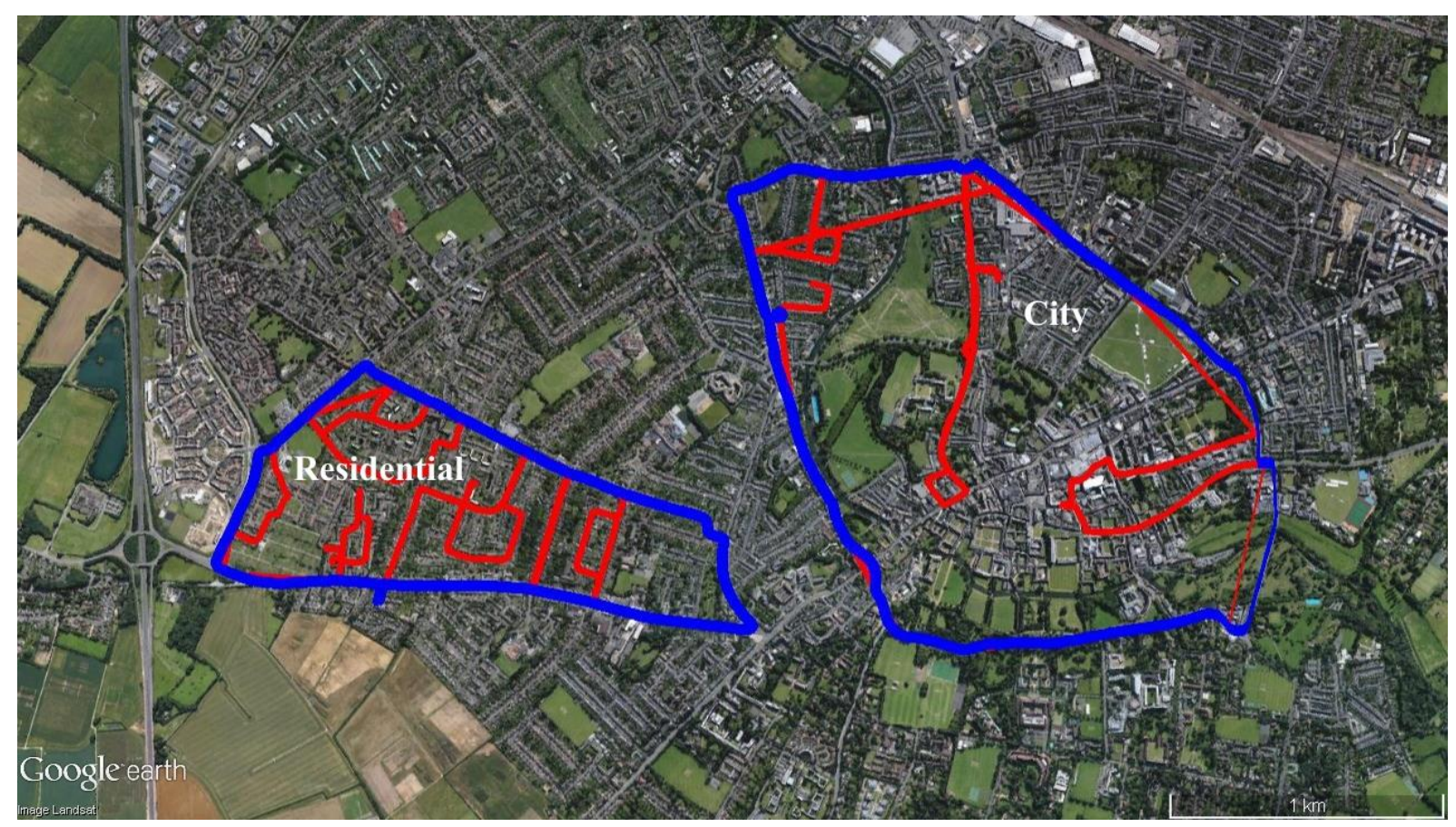

Figure 2. Cambridge area. Two regions in Cambridge, the United Kingdom, with car measurements along ring (blue) and inner (red) roads. "Inner" measurements outside the area demarcated by the ring road were removed from the inner data set (I).

Along each route, the measurement samples were pooled in tiles of 35 by 35 metres (m) (i.e., a number of 816 tiles per $\mathrm{km}^{2}$ ) from a grid superimposed on each area. At $30 \mathrm{~km} / \mathrm{h}$, per tile crossing 2 to 3 measurements were obtained per tile, and the arithmetic mean was chosen to represent the power density at the centre of that tile. For each area and signal, the results of each route were stored in separate data sets: inner $(\boldsymbol{I})$ and $\boldsymbol{r i n g}(\boldsymbol{R})$. Before further analysis, supposedly inner measurement data that coincided 
with the ring trajectory or were outside the area demarcated by $\boldsymbol{R}$ were removed from $\boldsymbol{I}$. It should further be noted that all distances (such as $d_{\text {area }}$ and $d_{\text {inner }}$ in Table 1) mentioned in this paper are calculated between the centres of the considered tiles. The minimum distance achievable between two points is thus $35 \mathrm{~m}$.

\subsection{Modelling}

To determine the specific knowledge deducible on the RF-EMF exposure in a certain geographical area using the aforementioned type of measurement data, we interpolated the power density between the centre points on the 35 by $35 \mathrm{~m}$ grid bounded by the ring roads (Figures 1 and 2), and compared (1) the distribution of the interpolated values with the distribution of the inner-area measurements (I) (Section Results - Grid Statistics), and (2) the interpolated with the measured power densities at inner-area measurement locations (Section Results - Validation).

For the interpolation, ordinary Kriging was used - as the power density is lognormally distributed, we first log-transformed the measurements - and fitted a spherical variogram model with nugget 0 to the samples. Our starting point in each area was the ring data set, $\boldsymbol{R}$. Minimal models, without additional inner data, are further denoted as $M_{R}$. Then, randomly selected $\boldsymbol{I}$ data samples were gradually added to upgrade the models, always retaining a random $25 \%$ subset of $\boldsymbol{I}$ containing for validation (hence, the maximum models, denoted as $M_{m}$, were built using all available $\boldsymbol{I}(75 \%)$ and $\boldsymbol{R}$ data.

To account for the uncertainty due to the limited number of samples (between 170 and 386 for interpolation and 23 to 47 for validation), we repeated each interpolation stage 200 times - each run, a new variogram model was fitted to the data - using random subsets of $\boldsymbol{I}$ for interpolation (max. 75\%) and validation (25\%), and averaged the interpolation and validation results (Tables 3 and 4) over all runs.

\section{Results}

\subsection{Measurements}

A summary of the measurements performed in the considered areas (aggregated per tile) can be found in Table 2. A higher power density was obtained for GSM900 (a geometric average of $60.6 \mu \mathrm{W} / \mathrm{m}^{2}$ $(0.15 \mathrm{~V} / \mathrm{m})$ within the inner areas, and $122 \mu \mathrm{W} / \mathrm{m}^{2}(0.21 \mathrm{~V} / \mathrm{m})$ on the enveloping ring trajectories $)$ compared to GSM1800 $\left(32.9-40.9 \mu \mathrm{W} / \mathrm{m}^{2}\right.$ or $\left.0.11-0.12 \mathrm{~V} / \mathrm{m}\right)$. The geometric standard deviation ranges between 2.80 and 8.40 , and is on average about 5 , with no apparent difference between signals and outer and inner measurements. In most cases, a large difference in power density is observed between ring and inner area. The highest discrepancy is found in Amersfoort-City, with a median power density on the ring of approximately 20 (GSM900) to 40 (GSM1800) times higher than within the inner area. In the other regions, the difference in median power density is at most a factor 2. 


\begin{tabular}{|c|c|c|c|c|c|c|c|c|}
\hline \multirow[b]{2}{*}{ Area } & \multicolumn{4}{|c|}{ INNER } & \multicolumn{4}{|c|}{$R I N G\left(\mu W / m^{2}\right)$} \\
\hline & $\underset{\left(\mu W / \mathbf{m}^{2}\right)}{\operatorname{Min} .}$ & $\underset{\left(\mu W / m^{2}\right)}{\text { Avg. }}$ & $\begin{array}{c}\text { Sd. } \\
(-)\end{array}$ & $\begin{array}{c}\text { Median } \\
\left(\mu W / \mathbf{m}^{2}\right)\end{array}$ & $\underset{\left(\mu W / \mathbf{m}^{2}\right)}{\operatorname{Min} .}$ & $\underset{\left(\mu W / \mathbf{m}^{2}\right)}{\text { Avg. }}$ & $\begin{array}{c}\text { Sd. } \\
(-)\end{array}$ & $\begin{array}{r}\text { Median } \\
\left(\mu W / m^{2}\right)\end{array}$ \\
\hline \multicolumn{9}{|l|}{ GSM900 } \\
\hline Amersfoort-City & $2.32-1862$ & 30.8 & 3.66 & 28.1 & $36.3-4433$ & 404 & 2.80 & 474 \\
\hline Amersfoort-Industrial & $16.4-2410$ & 221 & 3.68 & 235 & $4.55-2692$ & 155 & 5.15 & 223 \\
\hline Amersfoort-Residential & $1.03-287$ & 16.7 & 4.14 & 12.8 & $3.43-2133$ & 35.5 & 5.22 & 20.0 \\
\hline Cambridge-City & $0.91-2296$ & 23.2 & 6.22 & 18 & $0.44-3940$ & 10.8 & 7.22 & 7.85 \\
\hline Cambridge-Residential & $0.41-1619$ & 8.76 & 7.03 & 4.99 & $0.31-631$ & 3.44 & 4.89 & 2.71 \\
\hline Average & $4.21-1695$ & 60.6 & 4.95 & 59.8 & $9.01-2766$ & 122 & 5.06 & 146 \\
\hline \multicolumn{9}{|l|}{ GSM1800 } \\
\hline Amersfoort-City & $0.44-1069$ & 4.14 & 3.29 & 4.05 & $1.36-2452$ & 112 & 4.34 & 160 \\
\hline Amersfoort-Industrial & $6.44-3115$ & 138 & 3.80 & 148 & $3.38-1117$ & 65.1 & 4.29 & 71.8 \\
\hline Amersfoort-Residential & $0.02-143$ & 0.97 & 4.85 & 0.87 & $0.08-545$ & 1.80 & 8.40 & 1.54 \\
\hline Cambridge-City & $0.61-8952$ & 15.3 & 6.74 & 10.5 & $0.44-1405$ & 12.7 & 4.86 & 11.0 \\
\hline Cambridge-Residential & $0.35-662$ & 6.17 & 5.87 & 4.29 & $0.47-1394$ & 10.3 & 5.83 & 6.13 \\
\hline Average & $1.57-2788$ & 32.9 & 4.91 & 33.5 & $1.15-1983$ & 41.9 & 5.54 & $\mathbf{5 0}$ \\
\hline
\end{tabular}

Table 2: Power density measurements (aggregated per tile) of GSM900 (900 MHz band) and GSM1800 (1800 MHz band)

[Bolte et al., 2016]. (Avg. = the geometric mean; Sd. = the geometric standard deviation.)

\subsection{Modelling}

As expected from the dissimilarity between $\boldsymbol{R}$ and $\boldsymbol{I}$ power density distributions (Table 2), the ring-only models $M_{R}$ seem incapable of accurately predicting the inner-area power density distribution (Table 3), even more so for GSM1800 than for GSM900. However, disregarding the huge Amersfoort-City errors, the relative error in the prediction of the median power density lies between $-3.6 \mathrm{~dB}(-57 \%)$ and $+2.7 \mathrm{~dB}$ $(+87 \%)$; in comparison, the measurement uncertainty of a typical spot measurement setup is $+/-3 \mathrm{~dB}$ (Joseph et al., 2010b).

The trend observed when comparing grid statistics continues in the analysis of the validation (Table 4). Considering the overall sparsity of data, the ring-only models $M_{R}$ perform pretty well. Again disregarding Amersfoort-City, we found correlation parameters ranging between bad and moderate as well as low relative errors (always positive, and less than $+2 \mathrm{~dB}$ ), with the best scoring models in both Residential areas $(\rho=0.7-0.8, \kappa=0.5)$. 


\begin{tabular}{|c|c|c|c|c|}
\hline \multirow[b]{2}{*}{ Area } & \multicolumn{4}{|c|}{$M_{R}$ prediction } \\
\hline & $\underset{\left(\mu \mathrm{W} / \mathbf{m}^{2}\right)}{\operatorname{Min} .}$ & $\underset{\left(\mu W / \mathbf{m}^{2}\right)}{\text { Avg. }}$ & $\begin{array}{c}\text { Sd. } \\
(-)\end{array}$ & $\begin{array}{c}\text { Median } \\
\left(\mu W / m^{2}\right)\end{array}$ \\
\hline \multicolumn{5}{|l|}{ GSM900 } \\
\hline Amersfoort-City & $47.5-2980$ & 285 & 1.72 & 306 \\
\hline Amersfoort-Industrial & $7.08-1656$ & 157 & 2.75 & 169 \\
\hline Amersfoort-Residential & $5.10-1045$ & 25.5 & 2.87 & 21.1 \\
\hline Cambridge-City & $0.69-1987$ & 10.3 & 3.50 & 8.43 \\
\hline Cambridge-Residential & $0.36-385$ & 3.91 & 3.45 & 2.94 \\
\hline Average & $12.1-1611$ & 96.3 & 2.86 & 101 \\
\hline \multicolumn{5}{|l|}{ GSM1800 } \\
\hline Amersfoort-City & $2.15-825$ & 48.2 & 3.42 & 54.0 \\
\hline Amersfoort-Industrial & $5.46-464$ & 67.1 & 1.68 & 64.0 \\
\hline Amersfoort-Residential & $0.11-263$ & 1.66 & 3.92 & 1.63 \\
\hline Cambridge-City & $1.21-397$ & 10.4 & 2.18 & 10.6 \\
\hline Cambridge-Residential & $0.93-413$ & 9.09 & 2.99 & 7.69 \\
\hline Average & $1.97-472$ & 27.3 & 2.84 & 27.6 \\
\hline
\end{tabular}

Table 3: GSM900 and GSM1800 power density distributions of the ring-only interpolation models $M_{R}$, averaged over 200 interpolation runs. Predicted values are assigned to the grid tile centres. (Avg. = the geometric mean; Sd. = the geometric standard deviation.)

\begin{tabular}{lccccc}
\hline & $\boldsymbol{\rho}$ & $\boldsymbol{\kappa}$ & \multicolumn{1}{c}{ sensitivity } & specificity & $\boldsymbol{\Delta}_{\text {rel }}$ \\
GSM900 & & & & & \\
Amersfoort-City & $0.17(0.18)$ & $0.03(0.16)$ & $0.04(0.14)$ & $0.90(0.01)$ & $18.3(3.88)$ \\
Amersfoort-Industrial & $0.61(0.13)$ & $0.24(0.14)$ & $0.29(0.22)$ & $0.92(0.03)$ & $0.35(0.25)$ \\
Amersfoort-Residential & $0.81(0.04)$ & $0.52(0.11)$ & $0.41(0.20)$ & $0.93(0.02)$ & $0.79(0.20)$ \\
Cambridge-City & $0.70(0.10)$ & $0.36(0.15)$ & $0.70(0.19)$ & $0.96(0.02)$ & $-0.03(0.14)$ \\
Cambridge-Residential & $0.77(0.05)$ & $0.46(0.11)$ & $0.38(0.21)$ & $0.94(0.02)$ & $-0.00(0.13)$ \\
Average & $\mathbf{0 . 6 1}$ & $\mathbf{0 . 3 1}$ & $\mathbf{0 . 3 5}$ & $\mathbf{0 . 9 3}$ & $\mathbf{4 . 0 2}$ \\
GSM1800 & & & & & \\
Amersfoort-City & $0.31(0.18)$ & $0.17(0.18)$ & $0.06(0.16)$ & $0.91(0.02)$ & $30.3(7.95)$ \\
Amersfoort-Industrial & $0.21(0.15)$ & $0.16(0.13)$ & $0.05(0.13)$ & $0.89(0.02)$ & $0.37(0.35)$ \\
Amersfoort-Residential & $0.76(0.05)$ & $0.48(0.11)$ & $0.63(0.20)$ & $0.96(0.02)$ & $1.22(0.34)$ \\
Cambridge-City & $0.43(0.14)$ & $0.13(0.13)$ & $0.16(0.17)$ & $0.91(0.03)$ & $0.17(0.20)$ \\
Cambridge-Residential & $0.72(0.06)$ & $0.48(0.11)$ & $0.37(0.20)$ & $0.94(0.02)$ & $1.41(0.34)$ \\
Average & $\mathbf{0 . 4 9}$ & $\mathbf{0 . 3 1}$ & $\mathbf{0 . 2 7}$ & $\mathbf{0 . 9 2}$ & $\mathbf{6 . 4 0}$ \\
\hline
\end{tabular}

Table 4: Validation analysis of ring-only models $M_{R}$ (for GSM900 and GSM1800). All parameters are averaged over 200 interpolation runs and standard deviations are given between brackets. $(\rho=$ Spearman's correlation coefficient, $\kappa=$ Cohen's kappa (unweighted), and $\Delta_{\text {rel }}=$ relative error (modelled power density / measured power density - 1); cut-offs for calculation of $\kappa$, sensitivity and specificity are at 50th and 90th percentiles.) 


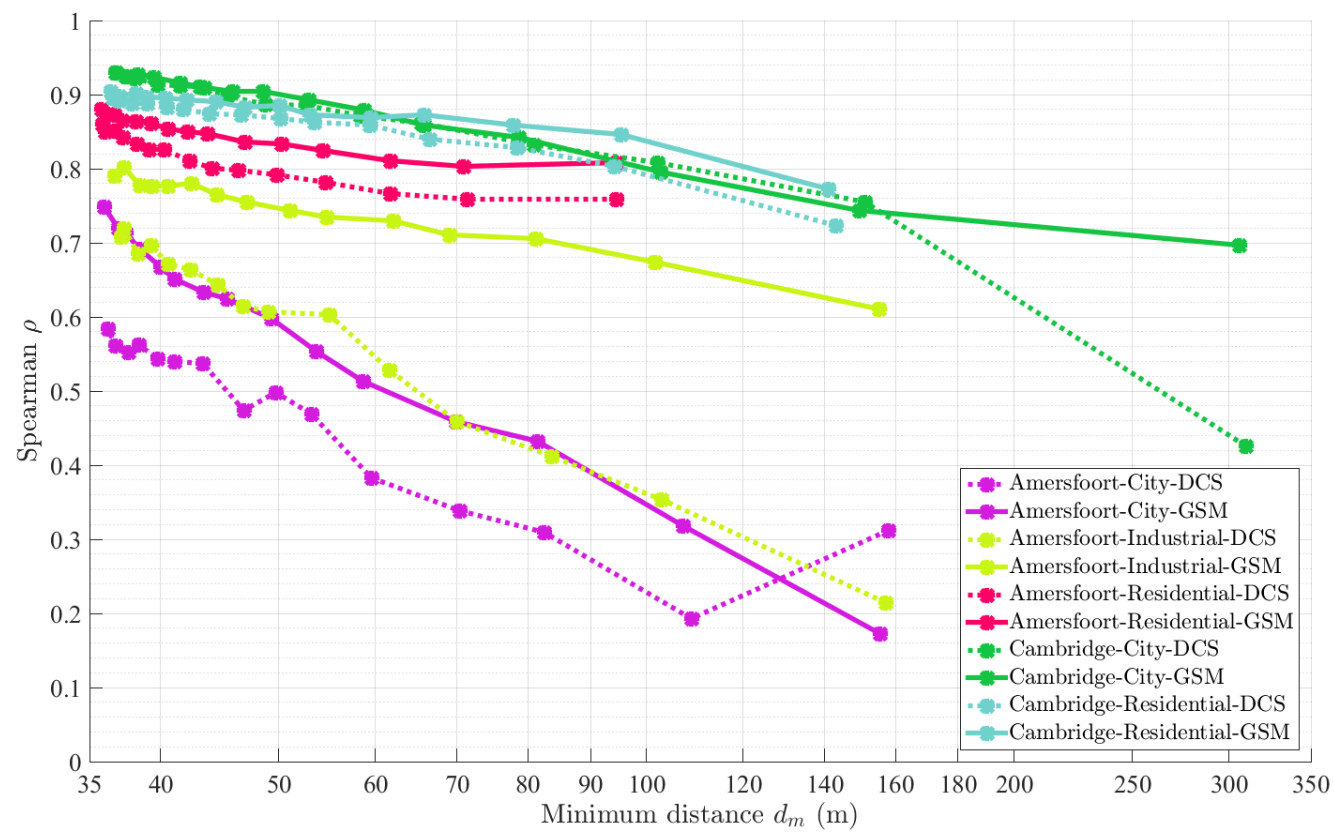

(a)

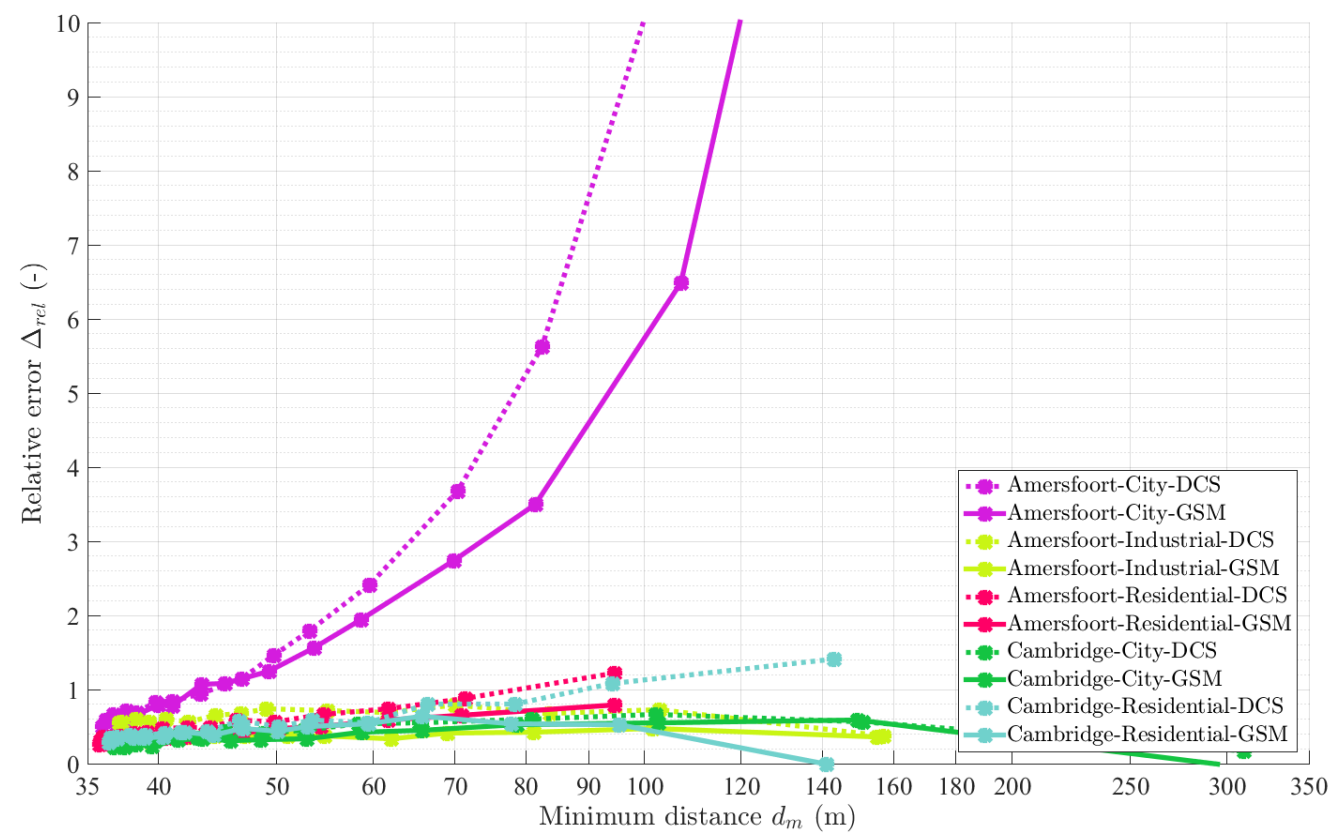

(b)

Figure 3: Kriging interpolation results. (a) Spearman's rank correlation coefficient $\rho$ and (b) the relative error $\Delta$ rel as a function of the minimum distance $d_{m}$ between validation and interpolation points. The outer right data points for each area are the $M_{R}$ results (no additional inner data added). When adding more inner data samples to the interpolation process, $d_{m}$ decreases. Other correlation parameters (e.g., Cohen's kappa) follow the same trend as shown in (a). (GSM = GSM900; DCS (Digital Cellular System) $=$ GSM1800). 
To determine the extent to which the predictive power of our interpolation models can be improved, we added measurements from the inner area ( $\boldsymbol{I}$ data set). The obvious way to analyse the influence of the inner data would be to show the evolution of the validation parameters as a function of the amount of inner samples (i.e., the percentage of inner area covered by measurements). However, due to the nature of the measurements, our validation data cannot be viewed completely independent of the kriged data, as they're on the same trajectory through the inner part of the area (Figures 1 and 2). Inner area coverage numbers would thus be useless as a measure. Furthermore, as a guideline for determining the type of enclosed area well suited to apply our methodology, the average minimum distance between the sample set and the interpolated area $\left(d_{m}\right)$ would be the most interesting measure. As shown in Figure 3, the additional inner samples boost the correlation parameters (between $+6 \%$, Amersfoort-Residential, and $+275 \%$, Amersfoort-City) and reduce the errors (up to $-2800 \%$ in Amersfoort-City), as was expected. At the minimum achievable $d_{m}$ (35 $\mathrm{m}$ or 1 tile) Spearman coefficients of $0.6-0.95$ (not shown: $\kappa=0.3-0.8)$ and relative errors of approximately $+50 \%(+1.8 \mathrm{~dB})$ are attained.

\section{Discussion}

The success of the ring-only models $M_{R}$ in the Residential areas can be attributed to their elongated shapes (Figures 1 and 2), allowing over the whole inside area interpolation based on two close-by sides. The Amersfoort-Industrial area, on the other hand, although an elongated trapezium shaped area as well, misses one long edge of its circumference (about one third) in its ring data set, which results in inferior interpolation models (Table 4). The moderate interpolation results for GSM900 can be explained by the coincidental similarity between ring and inner measurement distributions (Table 2).

The worst performing $M_{R}$ are the Amersfoort-City models. Amersfoort-City, a slightly flattened circle, misses a fifth of its circumference, but the completeness of the ring is clearly not the main factor. After closer inspection of the area, we believe its unsatisfactory performance is mainly caused by the limited sampling strategy. Amersfoort-City comprises a medieval centre with narrow streets and relatively high, dense buildings surrounded by a broad ring road (Figure 1). This ring road provides $\boldsymbol{R}$, while $\boldsymbol{I}$ consists mainly of a sole circle within the centre running parallel and is practically unconnected to the surrounding ring road, with a canal on the inner side and high buildings on the outer. These structural factors effectively shield the inner from the ring measurements, rendering them physically independent. Furthermore, the opposite is true for Cambridge-City, where the open character of the area mitigates the distance effect on the interpolation results (Table 1; its inner area is, on average, much farther away than in the other regions).

After adding inner-area measurements to the interpolation models, the difference in results between the considered areas can still be significant, even when the same $d_{m}$ is reached (Figure 3; e.g., AmersfoortCity vs. Cambridge-City), due to the factors explained in the previous section: a non-closed perimeter 
and structural factors. However, for comparable areas such as Amersfoort-Residential, Cambridge-City, and Cambridge-Residential the results tend to align well. While the initial $M_{R}$ results of Cambridge-City and to a lesser extent of Cambridge-Residential were meagre due to their respective sizes, the additional information from the inside area results in a steady increase in predictive power, and at an average $d_{m}$ of about $100 \mathrm{~m}$ (i.e., the initial $d_{m}$ of Amersfoort-Residential's $M_{R}$ ), the validation results are satisfactory $\left(\rho=0.8-0.9, \kappa=0.5-0.6, \Delta_{r e l}<3.5 \mathrm{~dB}\right)$ as well as remaining relatively unchanged by further additional inner sampling (Figure 3).

Furthermore, even with decreasing $d_{m}$, the models in Amersfoort-City and Amersfoort-Industrial (GSM1800) remain inadequate (Figure 3). For Amersfoort-Industrial, we believe that closing the ring would have solved the issue, as it would reduce the chance that a highly-exposed region on one side of the area is overlooked. For Amersfoort-City, however, the solution might be less straightforward. As mentioned in the previous section, structural factors (specifically for $M_{R}$ ) as well as the inner sampling strategy (for enhanced models) seem to be the main causes of the models' failure. We suggest that adding a sufficient amount of inner samples and performing the measurements in regions more physically connected to the ring would yield better interpolation results. Although, due to the very nature of these areas, it is unlikely that the results would be as good as in other, more open areas (e.g., both Residential areas).

On a side note, GSM1800 models seem to perform worse than GSM900 models, which might be due to its smaller wavelength. Moreover, for all model types, the average relative error is positive (Figure 3(b)), indicating an overestimation effect of the interpolation.

\subsection{Strengths and limitations}

In this paper we suggested an interpolation methodology to assess the RF-EMF exposure in large outdoor areas based on car-mounted measurements along the surrounding roads. The validity of such measurements in RF-EMF exposure assessment has previously been considered by Bolte et al. (2016). The aim here was to develop a means to assess the environmental RF exposure for larger areas.

In areas the size and shape of Amersfoort-Residential (with an average minimum distance of the encircled inner area to the edge of the area of less than $100 \mathrm{~m}$ ), our methodology can readily be applied when the measured ring route is closed. In this case, a Spearman coefficient of 0.8 , a $\kappa$ of 0.5 , and a specificity and sensitivity of respectively 0.94 and 0.50 can be attained while keeping the relative error below $3.5 \mathrm{~dB}$. In larger or non-ideal areas, additional inner measurements are recommended. Overall, we advise a maximum minimum distance $d_{m}$ of $100 \mathrm{~m}$ in the case of relatively open areas with mainly low, (semi-)detached buildings (Figure 3).

Compared to other RF-EMF exposure modelling techniques, the combination of our proposed method with the measurement method of Bolte et al. (2016) provides reasonable results. For example, after 
validation in five areas, the NISmap model for GSM900 by Beekhuizen et al. (2013) yielded Spearman coefficients of 0.71 to 0.85 and a relative error up to $-2.5 \mathrm{~dB}$, results that are none too far from the predictive capacity of our $M_{R}$. Further comparison can be found in Aerts et al. (2013a), in which Spearman coefficients of $0.54-0.86$, non-weighted $\kappa$ 's of $0.17-0.44$, sensitivities of $0.25-0.67$, and specificities of 0.88 - 0.96 are reported. However, while computer models such as NISmap are capable of estimating exposure at various heights, interpolation models based on car-mounted measurement data are limited to the height of the car roof, which is roughly $1.8 \mathrm{~m}$ above ground level.

The low sensitivities obtained for some $M_{R}$ (Table 4) suggest that highly-exposed regions within the area were missed by the ring measurements. As this is vital knowledge for any risk assessment, it is important that we take care to close the ring and to oversample in dense regions, both to reduce the chance of missing exposure hotspots.

The measurement data are subject to a number of potential uncertainties. Measurement uncertainties can arise due to the measurement setup and variations in the received power (e.g., due to temporal variation or small-scale fading). Also, when aggregating the measurements in tiles, some measurements might be erroneously assigned to a neighbouring tile, due to the combination of the inherent spatial inaccuracy of the GPS tracking system and the possible temporal discrepancy between GPS and RFeye systems, which can amount to a spatial inaccuracy of the measurement points of several meters. Neighbouring tiles thus become more similar, which would lead to smoothing and an overestimation of the correlation metrics. The latter uncertainty is an inherent issue to mobile measurements and is difficult to resolve. However, averaging over several measurements per tile, we believe its influence on our results is not significant.

Additionally, the limited number of samples used for interpolation and validation can weaken our conclusions (although, compared to other measurement-based studies, the numbers are quite high). We accounted for this by repeating the interpolation step a number of times and averaging the validation results. The inaccuracy of the results is described by the standard deviation (Table 4).

Although the two downlink signals we focused on, i.e., GSM900 and GSM1800, represent the dominant contributions to the outdoor RF exposure (Joseph et al., 2010a), there is an abundance of other RF-EMF signals that have to be taken into account for a full assessment of the outdoor exposure, such as Universal Mobile Telecommunications System (UMTS), Long Term Evolution (LTE), Frequency Modulation (FM) radio, and Wireless Fidelity (Wi-Fi). However, these signals can be assessed using the same method (though at different measurement settings (Bolte et al., 2016)), keeping in mind the possible inferior results for higher frequencies (important for UMTS and Wi-Fi). Moreover, as we presented here an outdoor exposure assessment methodology, we can only account for $12.5 \%$ of the total human exposure (Bolte and Eikelboom, 2012), as people typically spend $18 \mathrm{~h}$ or more indoors. However, in studies on effects on flora and fauna, the presented method will offer a more representative exposure assessment. 
The main strength of our methodology is the fact that mobile measurements spanning nationwide road grids are readily available on an annual basis. Thus, all that needs to be done is collect and interpolate the data, and, if necessary (such as in enclosed areas larger than $1 \mathrm{~km}^{2}$ or in very densely populated areas), perform some additional measurements, e.g., using a smart sampling scheme such as in Aerts et al. (2013a). While it might not be feasible to account for very large contiguous areas with scarce measurement coverage, it should be possible to use the presented methodology in longitudinal epidemiological studies in specific neighbourhoods, towns, or cities.

\section{Conclusions}

In this paper we proposed a methodology to assess environmental RF exposure at street level for larger areas using car-mounted measurements, data that are often available through nationwide spectrum regulation measurements. We concluded that our methodology can be equally successfully applied ( $\rho=0.8, \kappa=0.5, \Delta_{\text {rel }}<3.5 \mathrm{~dB}$ ) on a data set featuring a closed route around a decently sized area ( $\sim 1 \mathrm{~km}^{2}$, with an average minimum distance of the encircled area to the ring of less than $100 \mathrm{~m}$ ), containing mainly low, detached buildings. Additionally, in larger areas, additional sampling is advised, lowering the average minimum distance between the measurement samples and interpolated area to at most $100 \mathrm{~m}$, to achieve the same level of accuracy.

\section{Acknowledgements}

This research was funded by the Netherlands Organisation for Health Research and Development (ZonMw) under grant number 85500035. 


\section{References}

Aerts S, Deschrijver D, Joseph W, Verloock L, Goeminne F, Martens L, Dhaene T. 2013a. Exposure assessment of mobile phone base station radiation in an outdoor environment using sequential surrogate modeling. Bioelectromagnetics 34:300-311.

Aerts S, Deschrijver D, Verloock L, Dhaene T, Martens L, Joseph W. 2013b. Assessment of outdoor radiofrequency electromagnetic field exposure through hotspot localization using kriging-based sequential sampling. Environ. Res. 126:184-91.

Baan R, Grosse Y, Lauby-Secretan B, El Ghissassi F, Bouvard V, Benbrahim-Tallaa L, Guha N, Islami F, Galichet L, Straif K. 2011. Carcinogenicity of radiofrequency electromagnetic fields. Lancet Oncol. 12:624-626.

Baliatsas C, Bolte J, Yzermans J, Kelfkens G, Hooiveld M, Lebret E, van Kamp I. 2015. Actual and perceived exposure to electromagnetic fields and non-specific physical symptoms: an epidemiological study based on self-reported data and electronic medical records. Int. J. Hyg. Environ. Health 218:331-44.

Beekhuizen J, Vermeulen R, Kromhout H, Bürgi A, Huss A. 2013. Geospatial modelling of electromagnetic fields from mobile phone base stations. Sci. Total Environ. 445-446C:202-209.

Bolte JFB and Eikelboom T. 2012. Personal radiofrequency electromagnetic field measurements in the Netherlands: Exposure level and variability for everyday activities, times of day and types of area. Environ. Int. 48C:133-142.

Bolte JFB, Maslanyj M, Addison D, Mee T, Kamer J, Colussi L. 2016. Do car-mounted mobile measurements used for radio-frequency spectrum regulation have an application for exposure assessments in epidemiological studies? Environ. Int. 86:75-83.

Bürgi A, Frei P, Theis G, Mohler E, Braun-Fahrländer C, Fröhlich J, Neubauer G, Egger M, Röösli M. 2010. A model for radiofrequency electromagnetic field predictions at outdoor and indoor locations in the context of epidemiological research. Bioelectromagnetics 31:226-236.

CRFS Ltd. March, 2009. Capture of Spectrum Utilisation Information Using Moving Vehicles. CRFS_T00005B01_Ofcom_Final Report, Ofcom Tender No 32/2008. Cambridge, UK.

Cucurachi S, Tamis WLM, Vijver MG, Peijnenburg WJGM, Bolte JFB, de Snoo GR. 2013. A review of the ecological effects of radiofrequency electromagnetic fields (RF-EMF). Environ. Int. 51:11640.

Estenberg J, Augustsson T. 2014. Extensive frequency selective measurements of radiofrequency fields in outdoor environments performed with a novel mobile monitoring system. Bioelectromagnetics 35:227-30.

Frei P, Mohler E, Neubauer G, Theis G, Bürgi A, Fröhlich J, Braun-Fahrländer C, Bolte JFB, Egger M, Röösli M. 2009. Temporal and spatial variability of personal exposure to radio frequency electromagnetic fields. Environ. Res. 109:779-785.

Frei P, Mohler E, Bürgi A, Fröhlich J, Neubauer G, Braun-Fahrländer C, Röösli M. 2010. Classification of personal exposure to radio frequency electromagnetic fields (RF-EMF) for epidemiological research: Evaluation of different exposure assessment methods. Environ. Int. 36:714-720. 
International Commission on Non-Ionizing Radiation Protection (ICNIRP). 1998. Guidelines for limiting exposure to time-varying electric, magnetic, and electromagnetic fields (up to $300 \mathrm{GHz}$ ). Health Phys. 74:494-522.

Joseph W, Frei P, Röösli M, Thuróczy G, Gajšek P, Trček T, Bolte J, Vermeeren G, Mohler E, Juhász P, Finta V, Martens L. 2010a. Comparison of personal radio frequency electromagnetic field exposure in different urban areas across Europe. Environ. Res. 110:658-63.

Joseph W, Verloock L, Goeminne F, Vermeeren G, Martens L. 2010b. Assessment of general public exposure to LTE and RF sources present in an urban environment. Bioelectromagnetics 31:576579.

Schiphorst R, Slump CH. 2010. Evaluation of Spectrum Occupancy in Amsterdam Using Mobile Monitoring Vehicles. 2010 IEEE 71st Veh. Technol. Conf.:1-5.

Tell RA, Kavet R. 2014. A survey of the urban radiofrequency (RF) environment. Radiat. Prot. Dosimetry 162:499-507.

Viel J-F, Cardis E, Moissonnier M, de Seze R, Hours M. 2009. Radiofrequency exposure in the French general population: band, time, location and activity variability. Environ. Int. 35:1150-1154. 